\title{
Utilization and dominance of spatially separated cues as a function of water deprivation
}

\author{
JEROME S. COHEN and GREGORY HACHEY \\ University of Windsor, Windsor, Ontario, Canada N9B $3 P 4$
}

\begin{abstract}
Utilization and dominance of physically separate redundant-relevant cues were measured in albino rats under moderate $(18 \mathrm{~h} /$ day) or high $(23.5 \mathrm{~h} /$ day $)$ water deprivation. Cues were located on goal doors (brightness) and the floor (texture) in a two-choice discrimination apparatus. Three experiments were carried out with the following paradigms: incidental cue, redundant-relevant cue discrimination, and optional shift discrimination. Deprivation did not affect rate of acquisition of initial discrimination or cue dominance. Cue utilization, however, was inversely related to deprivation in redundantrelevant cue and optional shift discrimination tasks. These effects were limited, however. Overtraining on the redundant-relevant cue task eliminated the effect. Within the optional shift situation, only animals that initially acquired a texture cue discrimination were affected by deprivation. Other factors affecting cue utilization in these experiments were also described and discussed.
\end{abstract}

Several investigators have shown that increased food or water deprivation retards or prevents utilization of incidental or redundant-relevant cues in animals. Bruner, Matter, and Papanek (1955) found that only rats under moderate food-deprivation conditions were able to learn the relevance of an incidental response position task superimposed on an already learned brightness task. Cohen and Telegdy (1970) found a similar relationship for water deprivation when incidental foreground figures were superimposed on a background shade cue. Similar results have been generated from redundant-relevant cue discrimination tasks in which both cues are initially present. Moderately $(18 \mathrm{~h})$, as opposed to highly $(23.5 \mathrm{~h})$, water-deprived rats have been shown to be better able to utilize both size and shape (Cohen, Stettner, \& Michael, 1969) and line orientation and shade cues (Telegdy \& Cohen, 1971). Recently, Roy (1976) found that squirrel monkeys that had been fed prior to experimental sessions were more able to utilize both size and shape cues than animals that had been fed after each session. These findings support earlier notions that increased drive or arousal narrows or focuses attention to a few relevant cues (Easterbrook, 1959; Tolman, 1948).

In the above studies, both cues occupied the same physical space. In trying to determine if the above relationship would generalize to physically separated

\footnotetext{
Support for this study was supplied by The National Research Council of Canada, Grant APA-7450, and by monies given by the University of Windsor to its Department of Psychology. The authors wish to thank Dr. A. Kobasigawa, University of Windsor, for his valuable criticisms of the manuscript. This article is based on a paper delivered at the Canadian Psychological Association Convention, Toronto, 1976. Reprints may be obtained from: J. Cohen, Department of Psychology, University of Windsor, Windsor, Ontario, Canada N9B 3P4.
}

cues, Cohen and Sullivan (1973) had rats acquire a discrimination task with redundant-relevant side wall and goal door cues. Cohen and Tubaro (1974) performed an incidental cue experiment with similar dimensions. Unfortunately, water deprivation had no effect on an animal's ability to utilize such cues. It is possible that animals, regardless of deprivation level, find it difficult to attend to physically separated dimensions. Sutherland and Andelman (1967), for example, found that rats showed greater utilization of redundant relevant cues together (orientation and brightness of rectangles on the goal door) than separated (orientation of rectangles on goal doors and floor texture stimuli). It should be noted, however, that animals were generally unable to initially learn a discrimination from the side wall cue in Cohen and Tubaro's study (1974). Lack of any deprivation effects in our last two studies may have stemmed from the use of a particularly less salient side wall cue. In view of this very real possibility, it is necessary to examine the effects of water deprivation on cue utilization of physically separate, but easily noticeable, cues.

The present series of experiments is an extension of earlier research with relevant and physically separate cues (Cohen \& Sullivan, 1973; Cohen \& Tubaro, 1974). In the present experiments, however, highly $(23.5 \mathrm{~h})$ and moderately $(18 \mathrm{~h})$ water-deprived animals were presented with relevant goal-door brightness and decision-chamber floor texture cues. The use of a floor texture cue was based on research by Waller (1970), who found that rats could easily learn a discrimination task from cues located in such an area. Therefore, it was assumed that a floor texture cue would be easily noticed by our rats. Three experiments were carried out in the present study. In the first experiment, animals first acquired a goal 
door brightness or floor texture discrimination and then were exposed to an incidental floor texture or goal door brightness cue, respectively. In the second study, animals initially learned a discrimination in which both cues were redundant and relevant. An optional shift paradigm as described by Kendler (1971) was employed in the last experiment. Here animals acquired a relevant floor texture or door brightness cue in the presence of the other, irrelevant, cue. Then animals were presented with a redundantrelevant discrimination task in which the reinforcement outcomes for attributes of the initially relevant cue were reversed. In all three studies, tests for utilization of each cue separately and for cue dominance by opposed cues tests, as described by Kendler (1971), were used.

The present study departed from previous investigations in the use of a direct test for cue dominance and an optional shift paradigm. The optional shift task was used to gain additional information on the effects of water deprivation on cue utilization and cue dominance. Kendler (1971) has also argued that such a design is more appropriate than redundantrelevant or incidental cue paradigms for investigating cue dominance.

Predictions were based on the hypothesis of Easterbrook (1959) and Tolman (1948) that drive or arousal level is inversely related to incidental or redundant-relevant cue utilization. Moderately deprived animals were expected to demonstrate greater utilization of each cue than highly deprived animals in all three studies. No specific predictions were made for cue dominance as a function of deprivation.

\section{Method}

Although three separate experiments were conducted, similar methods were employed in each experiment. In the interests of conserving space, we shall describe the basic elements common to all three experiments and also describe the procedural differences in each study.

\section{Subjects}

Ninety-six male albino rats (Wistar strain) from the breeding colonies of the University of Windsor and Woodlyn Farms, Guelph, Ontario, were used. The animals were between 75 and 90 days old at the start of each experiment. During the course of the experiment, two animals failed to complete their experiment and were replaced by substitutes from the same breeding stock. All rats were individually housed throughout the course of each study. Each study contained 32 animals randomly divided into four groups of 8 animals each.

\section{Apparatus}

A two-choice discrimination apparatus, consisting of a covered start chamber $(18 \times 10 \times 10 \mathrm{~cm})$, a V-shaped decision chamber, and two goal chambers $(28 \times 21 \mathrm{~cm})$, was used. The decision and goal areas were $30 \mathrm{~cm}$ high. The decision chamber extended $47 \mathrm{~cm}$ from the startbox exit to the goal chambers. It was $12 \mathrm{~cm}$ wide at the startbox and $46 \mathrm{~cm}$ wide at the goal chamber. Entrance into either goal chamber was through one of two doors $(10 \times$ $10 \mathrm{~cm}$ ) at the end of the decision chamber. A clear $12-\mathrm{cm}$ plastic divider separated each door in the decision chamber. Operation of the apparatus occurred in the following manner. An animal was placed in the start chamber facing away from the decision chamber. As soon as it turned around and nosed the clear plastic guillotine door, the experimenter manually lifted the start chamber door. The animal was allowed to enter the decision chamber and could enter a goal chamber by pushing open one of the goal chamber doors. Liquid reinforcement $(10 \%$ sucrose solution) was provided by a sipping tube in the goal chamber. The apparatus contained removable decision chamber floors and goal doors. A floor panel covered the decision chamber floor, i.e., it was $47 \mathrm{~cm}$ in length and 12 to $45 \mathrm{~cm}$ wide from its narrow to wide end. The interior was painted mat gray and was illuminated by a $100-\mathrm{W}$ incandescent bulb situated over the entrance of the start box.

\section{Stimuli}

During training and testing phases of each experiment, removable black and white goal doors and decision floor panels were used. The left or right half of each floor panel contained either $1 / 2$-in. 18-ga wire-mesh hardware cloth or smooth wood. Both the wire-mesh and underlying wood surface were painted mat gray in order to minimize the visual characteristics of the texture stimuli. Floors completely covered with the wire mesh or uncovered (smooth) were used during testing trials.

\section{Procedure}

Shaping. Each experiment contained three basic phases: a shaping phase, a discrimination phase, and a testing phase. Shaping and discrimination phases were similar to those described by Cohen and Telegdy (1970). During shaping, all animals were maintained on a daily $21-\mathrm{h}$ water-deprivation schedule. They received water ad lib for $3 \mathrm{~h}$ in their home cages following each session. Throughout all experiments, the animals received food ad lib in their home cages. In the first stage, the animals were trained to open gray goal doors for a 10 -sec drink of $10 \%$ sucrose liquid per trial. By the end of this phase, the animals had experienced a daily session of 12 experimenter-corrected trials in which position of an unlocked and locked door randomly varied over trials according to Fellows' (1967) sequences. An experimenter-corrected trial consisted of replacing an animal into the start chamber immediately after it had approached and touched the locked door. An animal continued to be run in a trial until it approached and opened the unlocked door. Each animal received its next trial after all other animals had been run in the preceding trial. Since batches of 8-10 animals were run at a time, intertrial intervals ranged from 4 to $6 \mathrm{~min}$. These basic running procedures were continued into the actual discrimination and testing phases.

After being shaped to emit the instrumental response, the animals were randomly placed into high and moderate waterdeprivation groups. Highly water-deprived animals received daily 23.5-h deprivation and 0.5 -h free drinking periods in their home cages. Moderately water-deprived animals received $18 \mathrm{~h}$ of deprivation and $6 \mathrm{~h}$ of free drinking per day. The choice of these two deprivation levels was justified by the necessity of obtaining levels that would create different internal drive states without disrupting acquisition performance. Cohen, Stettner, and Michael (1969) reported that lower deprivation levels than the present moderate level were insufficient to maintain discrimination performance. Cohen and Oostendorp (1976) further showed that both deprivation levels created differential states of motivation as measured by approach tendencies to different incentives (sucrose vs water). In the present study, highly deprived animals displayed significantly less (Mann-Whitney $U$ test, $\mathrm{p}<.05$ ) weight gain $(48 \mathrm{~g})$ than moderately deprived animals $(72 \mathrm{~g})$ from their overall starting weights $(\overline{\mathrm{X}}=344 \mathrm{~g})$. Body weight differences can also be considered as an indication of differences in motivation (Bolles, 1975). All animals were maintained on their respective deprivation levels for 3 days in their home cages prior to actual discrimination training in order to adapt them to their deprivation schedules. 


\section{Discrimination Training}

Different discrimination training procedures were carried out in each experiment. A description of this phase for each experiment is presented.

Experiment 1: Incidental cue design. Within each deprivation group, half the animals were required to learn a simultaneous brightness discrimination based on goal door brightness and in the absence of differential floor panel stimuli. The other animals had to acquire a simultaneous floor texture discrimination in the presence of gray doors. Thus there were four separate deprivation-discrimination task groups (referred to as highbrightness, high-texture, moderate-brightness, moderate-texture), with $\mathrm{S}+$ and $\mathrm{S}-$ counterbalanced within each group. All animals received 12 experimenter corrected trials per day. Only the correct doors were unlocked. The position of $\mathrm{S}+$ varied randomly over trials according to Fellows' (1967) sequences in this and the following experiments. Acquisition of the discrimination task occurred when an animal made no more than two errors within two successive daily 12-trial sessions. This criterion was atso used in the following two experiments. Following acquisition, an animal received five sessions (60 trials) of incidental cue training. Brightness cue learners were exposed to a relevant floor texture cue along with the brightness cue, while floor texture cue learners received a relevant door brightness cue. Stimuli within each cue were perfectly correlated with reinforcement outcome. There were two possible combinations of stimuli: black door with smooth floor and white door with wire-mesh floor; white door with smooth floor and black door with wire-mesh floor. Presentation of these combinations and designation of $\mathrm{S}+$ and $\mathrm{S}$ - compound stimuli within each combination were counterbalanced within each group.

Experiment 2: Redundant-relevant cue design. Animals within each deprivation condition received the same stimulus compounds that animals in Experiment 1 experienced during their incidental cue phase. Half the animals within each deprivation (high or moderate) group were trained to acquisition criterion and then tested for cue utilization and cue dominance (referred to as the high-criterion and moderate-criterion groups). The other animals received five more overtraining sessions before being tested (referred to as the high-overtrained and moderate-overtrained groups). The overtrained groups received amounts of training closely equivalent to those given incidental-cue learners in the first experiment. The inclusion of overtrained groups permitted comparisons of cue utilization and cue dominance between animals of the first two experiments that had equivalent amounts of training.

Experiment 3: Optional shift design. Animals within each deprivation group initially acquired a simultaneous door brightness discrimination in the presence of an irrelevant floor-texture cue or a simultaneous floor-texture cue discrimination in the presence of an irrelevant door brightness cue. Thus there were four groups of animals labeled in terms of deprivation level and relevant cue: high-brightness, high-texture, moderate-brightness, moderate-texture. The irrelevant cue was so manipulated that both attributes occurred simultaneously, but were randomly paired with each attribute of the relevant stimulus on half the daily trials. After an animal reached criterion on this task, it was presented with a second, redundant-relevant, discrimination task. In that task, the irrelevant cue was now made relevant in that each attribute was consistently paired with reinforcement outcomes. The initially relevant cues continued to be relevant but the reinforcement properties of each attribute were reversed. As in the previous two experiments, all attribute combinations of redundant-relevant cues were counterbalanced within each group. Each animal was tested for cue utilization and cue dominance after it had acquired the second discrimination task.

\section{Testing}

Testing procedures were the same in all three experiments. It will be recalled that all animals received exposure to ledundant- relevant cues during the terminal portion of training. Such exposure continued into the testing sessions for the next 12 days. Interspersed among these regular redundant-relevant cue discrimination trials were the specific test trials. Every fourth trial was a test trial. On each daily session, an animal received a cue utilization test for door brightness, a cue utilization test for floor texture, and an opposed cues test for cue dominance. The order of presentation of each test trial was randomly varied over sessions so that animals received each test in each position for an equal number of times over the test phase. Both goal doors were unlocked on a test trial and choice of either door was reinforced. Sutherland and Holgate (1966) used a similar freechoice testing procedure to prevent test trials from becoming specific discrimination trials for the animals.

A brightness cue utilization test assessed an animal's ability to utilize the door brightness cue in the presence of the positive texture attributes. For example, if a black door and wire-mesh floor were the positive compound stimulus, black and white unlocked doors would be presented with a totally wire-meshed floor. In the texture cue test, animals had to choose between both texture attributes in the presence of both doors that contained the positive brightness attribute. In the above example, the animals would have received wire-mesh and smooth floor stimuli with both black doors. Opposed cues tests directly measured cue dominance or preference. All stimuli were presented simultaneously but paired oppositely from their usual relationship. For example, if black door with wire mesh and white door with smooth floor were the usual combinations, black door was now paired with smooth floor and white door with wire mesh in the opposed cues tests.

It will be noticed that cue utilization tests were not presented with the negative attribute of the other cue held constant, as had been done in previous studies (Sutherland \& Holgate, 1966; Telegdy \& Cohen, 1971). Such tests may have been too similar to opposed cues tests and could have affected responding to the latter. In other words, an animal could have learned to approach negative attributes of both stimuli from such cue utilization tests so that any initial cue dominance could have been masked. Therefore, we sought to make cue utilization tests dissimilar to opposed cues tests.

Throughout all phases of the experiments, floors vere wiped with a damp cloth periodically within and between sessions to prevent accumulation of other redundant visual and olfactory cues.

\section{RESULTS}

\section{Discrimination Acquisition}

Number of sessions and errors to criterion were measured in each experiment. Due to the small frequencies, $a \sqrt{X+0.5}$ transformation of number of sessions to criterion was carried out, as suggested by Winer (1971). However, for ease of comparison, mean number of sessions are presented rather than the transformed measures. Appropriate analysis of variance for both measures were carried out as dictated by the specific experimental design. Newman-Keuls tests for individual comparisons were also carried out where warranted.

In Experiment 1 (incidental cue learning), deprivation failed to have a significant effect on rate of acquisition (number of sessions), although type of initial discrimination did $[\mathrm{F}(1,28)=4.47, \mathrm{p}<.05]$. The animals acquired the brightness task slightly, but significantly, faster than the texture task ( 5 vs 6 
sessions, respectively). In terms of mean transformed scores, acquisition for brightness was $2.30(\mathrm{SD}=0.24)$ and for texture, $2.50(\mathrm{SD}=0.22$ ). Deprivation did interact significantly with type of task for error frequency, however $[F(1,28)=8.16, p<.01]$. This interaction was due to the fact that highly deprived animals required significantly fewer errors $(\overline{\mathrm{X}}=19$, $\mathrm{SD}=5.44$ ) than moderately deprived animals $(\overline{\mathrm{X}}=32, \mathrm{SD}=10.17)$ to acquire the brightness discrimination, whereas deprivation did not affect acquisition of the texture task (high $\bar{X}=29, \mathrm{SD}=$ 10.95; moderate $\bar{X}=23, S D=10.50$ ).

When animals were required to initially learn the redundant-relevant cue task (Experiment 2), deprivation had no significant effect on number of sessions to criterion $(\bar{X}=4)$ but did significantly affect error frequency $[F(1,28)=5.28, p<.05]$. Highly deprived animals made slightly, but significantly, fewer errors $(\bar{X}=14, S D=5.76)$ than moderately deprived animals $(\bar{X}=19, \mathrm{SD}=7.19)$.

In the third experiment, acquisition rates were measured for the initial task and optional shift task. No significant effects were found for deprivation, initial task, or between tasks (repeated factor) for errors to criterion. Animals made about as many errors in their initial discrimination as in the optional shift task $(\overline{\mathrm{X}}=30$ and $\overline{\mathrm{X}}=32$, respectively, $\mathrm{SD}=9.82$ ). When number of sessions was analyzed, however, animals required significantly more sessions to reach criterion on the first task than on the second $(\bar{X}=7$ vs $\bar{X}=5 ; \quad F(1,28)=22.00, \mathrm{p}<.01]$. Mean transformed scores were: Task $1=2.58$ vs Task $2=2.29, \mathrm{SD}=0.24$. No significant deprivation or discrimination task differences were found.

To summarize the acquisition results, deprivation did not affect number of sessions to criterion. A marginal effect on error frequency was found in the first two experiments, however. Increased deprivation led to fewer errors in acquiring the brightness task only (Experiment 1) and to slightly fewer errors in acquiring the redundant-relevant cue task (Experiment 2). Brightness was slightly easier to learn than texture, as measured by sessions to criterion in Experiment 1. When a variable irrelevant cue accompanied the relevant cue in Experiment 3, both cues were equally easy (or difficult) to learn. The animals required fewer sessions to learn the optionalshift task than their initial discrimination in Experiment 3.

\section{Cue Dominance}

It will be recalled that each animal received 12 test trials for each cue utilization test and for opposed cues tests. In Table 1, the data are presented in the form of proportion of "correct" choices for each cue utilization test and proportion of greater number of choices to the preferred cue on the opposed cues trials. For statistical analysis, proportion of choices for each animal was converted into a $2 \arcsin \sqrt{\mathrm{X}}$ transformation as suggested by Winer (1971). Separate analyses of variance were carried out for cue dominance (opposed cues tests) and cue utilization tests. The format of each analysis was determined by the specific design of each experiment. Two types of individual comparisons were also performed. In order to determine if animals were responding above a random level (.50) for each cue separately (cue utilization) or to one cue as opposed to the other (cue dominance) individual, singlesample $t$ tests were calculated. Transformed proportion scores were compared to a standard .50 (transformed) score within each cell. Pooled within-cell variances from each analysis of variance $(\mathrm{df}=28$, cue dominance; $\mathrm{df}=56$, cue utilization) were used for the error term rather than individual cell variances $(\mathrm{df}=7)$. Type I error due to multiple comparisons was reduced by decreasing $\alpha=.05$ to .025 for cue dominance and to .0125 for cue utilization, as specified by Mendenhall and Ramey (1973) (Ryan's procedure). Direct comparisons for amount of cue utilization and dominance comprised the second set of individual comparisons. A priori simple effects due to deprivation were calculated as dictated by hypotheses regardless of overall $F$ ratios from the analyses of variance. Other (post hoc) comparisons were carried out as determined by results from analyses of variance. For both a priori and post hoc tests, however, Newman-Keuls procedures were used in order to attenuate Type I error due to multiple comparisons.

Considering the cue dominance tests first, depriva-

Table 1

Proportion of Brightness or Texture Dominance Responses on Opposed Cues Tests and Proportion of Correct Responses on Cue Utilization Tests for Deprivation Groups in Each Experiment

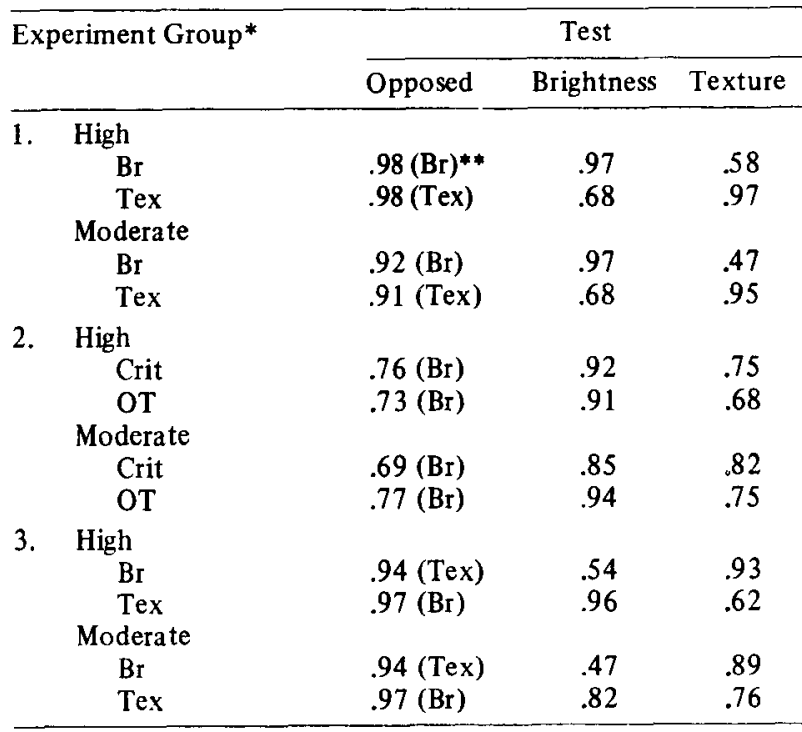

*Br-brightness cue learners; Tex-texture cue learners; Critcriterion; OT-overtrained.

* "Cue to which greater proportion of dominance responses were made: Br-brightness cue; Tex-texture cue. 
tion level had no significant effect on cue dominance in any of the three experiments. In the first study, brightness and texture cue learners displayed almost total dominance of their initially acquired cue on opposed cues tests. Such dominance measures were significantly greater than random (.50) responding $(\mathrm{p}<$.005). In Experiment 2, animals in all groups displayed dominance to the brightness cue over the redundant-relevant texture cue $(p<.005$ for both high-training groups and the moderate-overtrained group; $\mathrm{p}<.025$ for the moderate-criterion group). In Experiment 3 (optional-shift), all groups displayed significantly greater dominance to their initially irrelevant cue $(\mathrm{p}<.005)$.

\section{Cue utilization}

Findings from overall analyses of variance and simple-effects tests revealed that deprivation level did not consistently affect cue utilization. Other factors, such as type of cue test and type or amount of initial discrimination training, had substantial influence over amount of cue utilization, however.

In Experiment 1 (incidental cue), deprivation had no effect on cue utilization but type of cue test and initial cue acquisition had, as found in significant effects for cue test $[F(1,28)=5.77, p<.05]$ and the Initial Cue by Cue Utilization interaction $[F(1,28)=128.33, p<.01)$. All groups significantly utilized their initially acquired cue better than their incidental cue $(\mathrm{p}<.01)$. Both cue learning groups displayed similar levels of utilization of their respective initially acquired cues. Texture cue learners, however, made significantly more correct responses to their incidental brightness cue than brightness cue learners did to their incidental texture cue $(p<.01)$. This interaction was further brought out by the single-sample $t$ tests. All groups displayed utilization significantly above random responding (.50) to their initially learned cue $(p<.0005$ for each group), but only texture cue learners under either deprivation level were able to significantly utilize their incidental brightness cue $(\mathrm{p}<.005)$.

In Experiment 2 (redundant-relevant cues) all groups displayed significant utilization for each cue above random .50 responding $(p<.0005$ for each group). However, animals generally displayed better utilization of the brightness than the texture cue, as shown by a significant main effect for cue utilization $[F(1,28)=52.40, p<.01]$. Amount of training also significantly interacted with cue utilization $[F(1,28)=7.60, p<.05)$. The source of this interaction was due to the overtrained animals' displaying poorer utilization of the texture cue than criterion groups (deprivation level pooled) $(p<.05)$. Amount of training did not affect degree of utilization of the brightness cue.

Although overall $F$ ratios failed to reflect the influence of deprivation, significant simple effects for deprivation were found. Such effects were seen within (relative) rather than between (absolute) deprivation groups. Deprivation affected relative differences in cue utilization for animals trained to criterion but not for overtrained animals. Within the criterion-trained condition, moderately deprived animals showed similar utilization of each cue. Except for moderate-criterion animals, all other groups developed significantly greater utilization of the brightness cue than of the texture cue $(p<.01)$. Furthermore, only moderate-criterion animals developed significantly lower utilization of the brightness cue than their respective moderateovertrained animals $(\mathrm{p}<.05)$.

In Experiment 3 (optional-shift), both initial cue acquisition and deprivation level influenced cue utilization in an absolute as well as in a relative manner. Animals generally displayed reliable utilization of their nonreversed cue and generally showed near-random responding to their reversed cue. Deprivation did affect this relationship, however, for animals that had initially acquired the floor texture cue. Highly water-deprived animals failed to utilize their originally acquired texture cue, while moderately deprived animals displayed utilization of the texture cue to almost the same degree as to the brightness cue. A three-way analysis of variance revealed significant main effects for type of initial cue acquisition $[F(1,28)=5.78, p<.05)$ and cue utilization tests $[F(1,28)=7.64, \mathrm{p}=.01]$ and significant interactions of deprivation $[\mathrm{F}(1,28)=5.00, \mathrm{p}<.05]$ and type of initial cue acquisition $[F(1,28)=73.45$, $\mathrm{p}<.01]$ with cue utilization tests. An apparent triple interaction failed to reach significance $[F(1,28)=2.91$, $.05<\mathrm{p}<.10$ ], which may have been obscured by the relatively large Initial Cue by Cue Test interaction. Separate analyses for each initial cue acquisition group, however, did uncover a significant deprivation effect only for the initial texture cue learners [Deprivation by Cue Utilization Tests interaction: $F(1,14)=10.75, p<.01$ ]. As expected, a significant main effect for cue utilization tests was found for each initial cue acquisition condition $[F(1,14)=23.13$ for initial brightness cue learners, $\mathrm{F}=50.43$ for initial texture cue learners, $\mathrm{p}<.01]$. For initial texture cue learners, only moderately deprived animals displayed similar amounts of utilization of both cues while highly deprived animals significantly utilized the brightness cue better than the texture cue $(p<.01)$. Furthermore, for the texture cue groups, moderately deprived animals responded correctly to each cue significantly above chance (.50), while highly deprived animals only did so to the brightness cue ( $p<.005$ for each case). Such moderately deprived animals also made significantly more correct responses to the texture cue than highly deprived animals $(p<.05)$. On the other hand, high-texture cue animals made slightiy, but significantly, more correct responses to the brightness cue than their moderate counterparts $(p<.05)$. 
For initial brightness cue learners, deprivation had no influence on cue utilization. Both deprivation groups utilized the texture cue significantly better than the brightness cue $(p<.01)$. Only the texture cue was correctly utilized above random responding (.50) by both groups $(\mathrm{p}<.0005)$.

\section{CONCLUSIONS AND DISCUSSION}

The prediction that decreased water deprivation would increase utilization of spatially separated redundant-relevant cues was partially validated in the present study. Deprivation level did not affect utilization of an incidental cue in Experiment 1. In Experiment 2, deprivation had a marginal effect in that, although animals were generally able to utilize both cues, only moderately deprived animals trained to criterion failed to display better utilization of the brightness than of the texture cue. These results were apparently due to decreased responding to the brightness cue, since overtraining led to higher responding to this cue in moderately deprived animals. Although deprivation affected cue utilization in a more absolute manner in Experiment 3, this effect was confined to animals that initially acquired floor texture discrimination. Within this task group, highly deprived animals were only able to utilize the door brightness cue while moderately deprived animals correctly responded to each cue. Although these results are limited, they indicate that the inverse relationship between cue utilization and drive originally proposed by Tolman (1948) and expanded upon by Easterbrook (1959) is not confined to situations in which redundant-relevant cues occupy the same physical space. Previous failures to obtain such a relationship with physically separated cues (Cohen \& Sullivan, 1973; Cohen \& Tubaro, 1974) were probably in part due to the use of a less noticeable peripheral side-wall cue than the present floortexture cue.

Findings in the present study also indicate that cue dominance on opposed cues tests was unaffected by deprivation. Acquisiton of initial discrimination tasks was also generally unaffected by deprivation (number of trials to criterion). Only in Experiments 1 (only for brightness discrimination) and 2 did highly deprived animals make fewer errors than moderately deprived animals in acquiring the discrimination. Type of discrimination also affected rate of acquisition. Door brightness was slightly easier to acquire than floor texture in Experiment 1. The optional shift tasks required slightly less time to learn than the original task in Experiment 3.

The limited verification of our expectations may provide important information on the operation of deprivation in cue utilization. For example, the absence of deprivation effects on incidental cue utilization in Experiment 1 is inconsistent with earlier reports (Bruner et al., 1955; Cohen \& Telegdy, 1970). The present experiment differed from earlier research, however, in that our animals received far more exposure to the incidental cue prior to testing (60 vs $12-20$ trials). Mackintosh (1975a, b) recently proposed and experimentally verified that rats are more likely to exhibit associations between an incidental cue and reinforcement during initial exposure to the redundant cue in blocking paradigms. With continued exposure, however, these animals may either learn to ignore such stimuli and or increase attention to previously learned stimuli as sources of information. It would appear, in the present case, that decreased deprivation limited initial attention to the originally acquired door cue but that such attention increased independently of attention to the incidental cue with continued exposure.

As seen in the third study, initial type of discrimination interacted with deprivation in cue utilization. The results from the first two studies suggest a possible explanation for the specific type of interaction that was found. It will be recalled that animals that had acquired the texture cue discrimination in the first study were able to utilize the incidental brightness cue while brightness cue learners apparently did not pick up the relevance of the texture cue. While animals generally were able to utilize both cues in Experiment 2, they apparently preferred the door cue as a source of information about reinforcement on opposed cues trials. Stated in Mackintosh's (1975a) scheme, the animals tended to rely on the door cue as a "more valid" predictor for reinforcement. That such should be the case is hardly surprising when the attentional requirements of the basic instrumental response of opening a goal door are considered. All animals would have to attend to the general position and location of goal doors in order to avoid missing them and colliding into the surrounding wall. Animals that learned a specific door discrimination would not need to attend to and could ignore any redundant floor cues, but animals in the floor-texture group would have to also attend to the goal doors. The latter group, therefore, would be more likely to pick up the addition of any specific door stimuli than the former would any specific floor-texture stimuli in Experiment 1. Since such attentional differences did not hinder the acquisition of discrimination based on the nonreversed cue during the optional shift phase in Experiment 3, we further speculate that such attentional differences also affected general strategies for acquiring new discrimination tasks. Specifically, brightness cue discriminators may have learned to ignore redundant cues while texture discriminators may have learned either to utilize or at least not to ignore such cues in new problems. Deprivation would be more likely to have the predicted effect within a situation, e.g., initial floor-texture task, in which animals were not predisposed to ignore other possible 
sources of relevant information. This proposition would explain why, in Experiment 3, moderately deprived animals displayed greater cue utilization than highly deprived animals only if they had first acquired a texture cue discrimination. Bruner, Mandler, O'Dowd, and Wallach (1958) have also invoked the concept of a "generic principle" being more easily acquired under decreased deprivation. Their concept was based on findings that animals under lower food deprivation were better able to transfer a general response alternation sequence in a reversal shift problem than highly food deprived animals.

The present research may also provide important information on the general nature of cue utilization and selection. For example, the animals in Experiment 2 generally maintained high levels of responding to the brightness cue over training conditions but decreased utilization of the texture cue with overtraining. These results are contrary to a selective attention account (Sutherland \& Mackintosh, 1971) which predicts that decreased attention to one cue should be accompanied by increased attention to the other. Mackintosh (1975a), however, maintains that the strength of attention to each cue is independent of the other but that, with continued exposure, an organism will learn to ignore that cue which predicts reinforcement less efficiently. Our present findings generally support this notion.

Of further interest is the fact that strong cue dominance on opposed-cue tests did not preclude utilization of the less dominant cue, as clearly evidenced in Experiment 2. These results replicate earlier findings of utilization of both dominant color and nondominant form cues in rats (Kendler; Basden, \& Bruckner, 1970). Again, such findings are difficult to reconcile with the selective attention approach (Sutherland \& Mackintosh, 1971). It is noteworthy that animals that developed greater cue dominance in Experiments 1 and 3 than in Experiment 2 also displayed larger differences in utilization between such cues, as would be predicted by selective attention theory. However, animals that developed strong dominance to the floor-texture cue were still able to use the goal-door brightness cue. Such was not the case for the texture cue when the brightness cue was dominant. As discussed above, it would appear that orientation to a general area of reinforcement (goal doors) that contain incidental cues may be sufficient for acquisition of the relevance of such cues. Indeed, when redundantrelevant cues occupy the same physical space as in Kendler et al.'s (1970) study, even greater utilization of incidental cues might be expected since orientation to both cues would occur simultaneously. Therefore, the results in Experiments 1 and 3, as well as in Experiment 2 , are contrary to selective attention theory but support a more general attention model of cue utilization (Mackintosh, 1975a; Thomas, 1970).

\section{REFERENCES}

Bolles, R. C. Theory of motivation (2nd ed.). New York: Harper and Row, 1975.

Bruner, J. S., Mandler, J. M., O'Dowd, D., \& Warlach, M. A. The role of overlearning and drive level in reversal learning. Journal of Comparative and Physiological Psychology, 1958, 51, 607-613.

Bruner, J. S., Matter, J., \& Papanek, M. L. Breadth of learning as a function of drive level and mechanization. Psychological Review, 1955, 62, 1-10.

Conen, J. S., \& Oostendorp, A. Incentive preference under two levels of water deprivation in the rat. Bulletin of the Psychonomic Society, 1976, 8, 381-384.

Cohen, J. S., Stettane, L. J., \& Michael, D. J. Effect of deprivation level on span of attention in a multi-dimension discrimination task. Psychonomic Science, 1969, 15, 31-32.

Cohen, J. S., \& Sullivan, B. Effects of drive level on cue utilization of spatially separated redundant-relevant cues. Bulletin of the Psychonomic Society, 1973, 1, 455-457.

Cohen, J. S., \& Telegdy, G. A. Effect of drive level on habit strength in a discrimination task. Psychonomic Science, 1970 , 19. $27-29$.

CoHen, J. S., \& Tubaro, G. Effects of thirst drive on cue utilization and cue dominance of spatially separate cues in albino rats. Bulletin of the Psychonomic Society, 1974, 4, 451-453.

EASTERBRoOK, J. A. The effect of emotion on cue utilization and the organization of behavior. Psychological Review, 1959, 66, 183-201.

Fellows, B. Chance stimulus sequences for discrimination tasks. Psychological Bulletin, 1967, 67, 87-92.

KENDleR, T. Continuity theory and cue dominance. In $\mathbf{H}$. $\mathbf{H}$. Kendler \& J. T. Spence (Eds.), Essays in neo-behaviorism. New York: Appleton-Century-Crofts, 1971. Pp. 237-264.

Kendler, T. S., BAsden, B. H., \& BRuckner, J. B. Dimensional dominance and continuity theory. Journal of Experimental Psychology, 1970, 83, 309-318.

Mackintosh, N. J. A theory of attention: Variations in the associability of stimuli with reinforcement. Psychological Review, 1975, 82, 276-298. (a)

Mackintosh, N. J. Blocking of conditioned suppression: Role of the first compound trial. Joumal of Experimental Psychology, 1975. 1, 335-345. (b)

Mendenhall, W., \& Ramey, M. Statistics for psychology. Belmont: Wadsworth, 1973

Roy, M. A. Effects of drive level on the degree of cue control by squirrel monkeys. Psychological Reports, 1976, 38, 1155-1162.

Sutrerland, N. S., \& Andelman, L. Learning with one and two cues. Psychonomic Science, 1967, 7, 107-108.

Sutherland, N. S., \& Holgate, V. Two-cue discrimination learning in rats. Joumal of Comparative and Physiological Psychology, 1966, 61, 198-207.

Sutherland, N. S., \& Mackintosh, N. J. Mechanisms of animal discrimination learning. New York: Academic Press, 1971.

Telegdy, G. A., \& CoHen, J. S. Cue utilization and drive level in albino rats. Journal of Comparative and Physiological Psychology, 1971, 75, 248-253.

Thomas, D. R. Stimulus selection, attention, and related matters. In J. H. Reynierse (Ed.), Current issues in animal learning. Lincoln: University of Nebraska Press, 1970. Pp. 311-356.

Tolman, E. C. Cognitive maps in rats and men. Psychological Review, 1948, 55, 189-208.

WALLER, T. G. Effect of irrelevant cues on discrimination acquisition and transfer in rats. Journal of Comparative and Physiological Psychology, 1970, 73, 447-480.

WINER, B. J. Statistical principles in experimental design (2nd ed.). New York: McGraw-Hill, 1971. 